\title{
ALOE EMODIN ENHANCES TAMOXIFEN CYTOTOXICITY EFFECT ON ER $\alpha$-POSITIVE BREAST CANCER CELLS, MCF-7, THROUGH DOWNREGULATION OF MEK1 AND MEK2
}

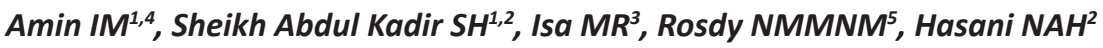 \\ 1 Institute for Medical Molecular Biotechnology (IMMB), Faculty of Medicine, Universiti Teknologi MARA (UiTM), Selangor \\ 2 Department of Biochemistry and Molecular Medicine, Faculty of Medicine, Universiti Teknologi MARA (UiTM), Selangor \\ 3 Department of Population Health and Preventive Medicine, Faculty of Medicine Universiti Teknologi MARA (UiTM), \\ Selangor \\ 4 Centre of Preclinical Sciences Studies, Faculty of Dentistry, UiTM, Selangor \\ 5 Centre of Oral and Maxillofacial Diagnostic and Medicine Studies, Faculty of Dentistry, UiTM, Selangor
}

\section{Correspondence:}

Assoc. Prof. Dr Narimah Abdul Hamid Hasani

Faculty of Medicine, Sungai Buloh Campus, Universiti Teknologi MARA, Jalan Hospital, 47000, Sg. Buloh, Selangor, Malaysia Telephone number: $+6017-9092341$

Fax number: $+603-61267073$

Email:drnarimah@salam.uitm.edu.my

\begin{abstract}
The positive response to tamoxifen in ER $\alpha$-positive breast cancer patients is usually of a short duration as many of the patients eventually develop resistance. Our preliminary results show that aloe emodin extracted from the leaves of the Aloe barbadensis Miller demonstrated a cytotoxicity that is selective to ER $\alpha$-positive breast cancer cells (MCF-7), but not to ER $\alpha$-negative breast cancer cells (MDA-MB-231) and to the control cells (MCF10A). The objective of this study was to test the hypothesis that aloe emodin may enhance the response of MCF-7 cells to treatment with tamoxifen. MCF-7 cells were treated with aloe emodin alone, tamoxifen alone or a combination of emodin and tamoxifen, at their respective $\mathrm{IC}_{50}$ concentrations and at different time points of 24 hours, 48 hours and 72 hours. The respective $\mathrm{IC}_{50 \text { s }}$ were the concentrations of aloe emodin and tamoxifen required to achieve $50 \%$ inhibition of the cells in the study. Cell viability and apoptosis were determined using trypan blue exclusion and DNA fragmentation assays, respectively. The involvement of RAS/MEKs/ERKs genes of MAPK signalling pathways with aloe emodin was determined using QuantiGene 2.0 Plex assay. Data was evaluated using the one-way ANOVA test. Our findings showed that aloe emodin enhanced the cytotoxicity of tamoxifen on MCF-7 cells through apoptosis by downregulation of MEK1/2 genes. Our research may provide a rational basis for further in vivo studies to verify the efficacy of a combination of aloe emodin and tamoxifen on the viability of ER $\alpha$-positive-breast cancer cells.
\end{abstract}

Keywords: Aloe emodin, tamoxifen, MAPK, MCF-7.

\section{Introduction}

Breast cancer is the most common cancer in women, with the highest prevalence among Asians including Malaysians $(1,2)$. A Malaysian cohort study from the year 2000 to 2005 showed that the overall 5-year survival rate of breast cancer patients among Malaysian women was lower compared to survival rates in developed nations (2). Nearly two thirds of all breast cancer patients expressed estrogen and progesterone receptors, responding with growth to these hormones (3). Estrogen stimulated the proliferation of estrogen receptor $\alpha(E R \alpha)$-positive breast cancer cells through the activation of the extracellular signal-regulated kinase (ERK) proliferation pathway (4). Tamoxifen is a selective estrogen receptor modulator (SERM) and directly competes with estrogen for receptor binding (5). It is an endocrine therapy of choice for treatment of advanced ER $\alpha$-positive breast cancer patients as it demonstrates a benefit when used alone and in combination with other chemotherapeutic agents (5). It reduces tumour recurrence, prolonging survival when administered as a post-operative adjuvant therapy in stages I and II of the disease $(5,6)$.

Deregulation of ERK pathway is seen in approximately one third of all human cancers (6). The ERK is one of three subfamilies of the mitogen-activated protein kinases (MAPKs). The others are c-Jun N-terminal kinase (JNK) and 
p38 kinase. The MAPKs pathways control the essential homeostatic balance between proliferation and death of cells in the fundamental development and maintenance of cellular processes (6). The ERKs and JNKs control cell proliferation and apoptosis respectively, whilst p38 is activated by inflammatory agents (6). The ERK pathway consisting of RAS/RAF/MEK/ERK is critical in transmitting proliferative extracellular signals such as growth factors or mitogens generated by cell surface receptors, to regulate gene expression and prevent apoptosis $(6,7)$. Abnormal ERK signalling is prevalent in most cancers as a consequence of increased expression or mutation of its upstream components (8). It may lead to uncontrolled cell proliferation, resistance to apoptosis, and resistance to chemotherapy, radiotherapy and targeted therapies (8). The activation of RAF/MEK/ERK pathway leads to phosphorylation of pro-apoptotic Bad, thus allowing antiapoptotic Bcl-2 to form homodimers and prevent apoptosis (9). It has been shown that the activation of RAF/MEK/ ERK cascade inactivates Caspase- 9 by phosphorylation thus blocking apoptosis (10). Increased expression of RAS/RAF/MEK/ERK pathway is associated with a poor prognosis in ER $\alpha$-breast cancer patients $(4,8)$. Activation of RAS pathway increases the sensitivity of ER $\alpha$ to low concentrations of estrogen and reduces tamoxifen efficacy $(11,12)$. Over expression of ERK1/2 is frequently seen in association with advanced stage of ovarian (13) and prostatic (14) cancers and their poor prognosis. There is a continuing effort in developing new strategies to enhance and prolong the efficacy of tamoxifen therapy. Aloe emodin (1, 8-dihydroxy-3-hydroxymethyl-anthraquinone) is found abundantly in Aloe barbadensis Miller leaves. It is phytoestrogenic due to its ability to limit the proliferation of $E R \alpha$-positive breast cancer cells by downregulating $E R \alpha$, thus suppressing $E R \alpha$ transcriptional activation. It has no effect on ER $\alpha$-negative cells proliferation (15). As analogues, synthetic estrogen-like compounds such as tamoxifen competes for estrogen binding sites of ER $\alpha$ and therefore inhibits the growth of ER $\alpha$-dependent breast cancer cells (12). These analogues exhibit side effects that increase the risk of cancer development due to unselective estrogenic action (12). The estrogenic potency of natural phytoestrogens is generally lower than synthetic estrogens and they have fewer side effects (16). Aloe emodin and its isomer, emodin (1,3,8-trihydroxy-6-methylanthraquinone), also enhance the inhibitory effect of chemotherapeutic agents such as cisplatin, doxorubicin, 5-fluorouracil and tyrosine kinase inhibitor (STI 571) on Merkel cell carcinoma (MCC) and prostate cancer cells (DU-145) especially at low concentrations of drugs used $(17,18)$. Aloe emodin is slightly more potent compared to emodin (16). These findings provide the evidence to suggest that aloe emodin is a suitable candidate for use in combination with chemotherapeutic agents such as tamoxifen in the management of ER $\alpha$-positive breast cancer patients. Further investigation is required to determine the underlying signalling pathways of aloe emodin-induced cell death in MCF-7 cells.

\section{Methods}

\section{Treatments and reagents}

Aloe emodin ( $\geq 95 \%$ HPLC) and tamoxifen were each dissolved in dimethyl sulfoxide (DMSO) to prepare primary stocks of $50 \mathrm{mM}$. Each final working solution of aloe emodin and tamoxifen were diluted in culture media so that the final concentration of DMSO in cell culture was $<0.1 \%$ (GIBCO Invitrogen, USA). Aloe emodin, tamoxifen and DMSO were purchased from Sigma Chemical Co., USA. Disposable sterile consumables (Brand Axygen, USA) and chemicals used were of tissue culture grade.

\section{Cell lines and culture}

Both ER $\alpha$-positive and ER $\alpha$-negative breast cancer cells, MCF-7 and MDA-MB-231, respectively (American Type Culture Collection, USA) were cultured in complete RPMI 1640 media supplemented with $10 \%$ fetal calf serum and $1 \%$ of penicillin and streptomycin (GIBCO Invitrogen, USA). ER $\alpha$-negative non-transformed breast cells, MCF-10A (American Type Culture Collection, USA) were maintained in complete DMEM high glucose media supplemented with $5 \%$ horse serum, $20 \mathrm{ng} / \mathrm{ml}$ of EGF, $0.5 \mathrm{mg} / \mathrm{ml}$ of hydrocortisone, $10 \mu \mathrm{g} / \mathrm{ml}$ insulin and $1 \%$ of penicillin and streptomycin (GIBCO Invitrogen, USA). All cells were maintained as a monolayer up to $80 \%$ confluence in humidified atmosphere of $5 \% \mathrm{CO}_{2}$, at $37^{\circ} \mathrm{C}$ in $\mathrm{T} 25$ and T75 flasks (Orange Scientific, Belgium). All cell culture experiments were performed under sterile condition in tissue culture hood.

\section{Cell viability}

Cell viability was performed using trypan blue exclusion assay (Sigma Chemical Co., USA). Cells were seeded at optimized $4 \times 10^{5}$ in a 6 -well plate (Orange Scientific, Belgium) and treated with aloe emodin, tamoxifen and the combination of both at their respective $\mathrm{IC}_{50}$ concentrations obtained from WST-1 proliferation assay (Amin et al., 2013) for 24 hours, 48 hours and 72 hours in $5 \% \mathrm{CO}_{2}$ at $37^{\circ} \mathrm{C}$. Tamoxifen was used as positive control. Cells cultured in complete media with $0.1 \%$ of DMSO were used as negative control. Cell morphology was observed under an inverted light microscope (Olympus, Japan) at 100X magnification. An equal volume of cell suspension and $0.4 \%$ trypan blue dye were mixed thoroughly and allowed to stand for 5 minutes. From the sample mixture, $10 \mathrm{~mL}$ was added into the chamber port on the Countess Cell counting chamber slide (Invitrogen, Canada). Slides were inserted into the port and counted. A viable cell appeared as a bright centre with dark edges, while a dead cell was blue and without any bright centre (19).

\section{Cell apoptosis}

Cell apoptosis was determined using morphological evaluation with acridine orange (AO; Sigma Chemical Company, USA) and propidium iodide ( $\mathrm{PI}$; Sigma Chemical Company USA) dual staining technique. A total of $4 \times 10^{5}$ 
cells were seeded in a 6-well plate (Orange Scientific, Belgium) and treated with aloe emodin, tamoxifen and the combination of both at their respective $\mathrm{IC}_{50}$ concentrations for 48 hours and 72 hours in $5 \% \mathrm{CO}_{2}$ at $37^{\circ} \mathrm{C}$. Tamoxifen and untreated cells were used as positive and negative controls, respectively. After staining with $1 \mathrm{mg} / \mathrm{mL} A O$ and $1 \mathrm{mg} / \mathrm{mL}$ PI for 30 minutes, the fluorescent images were captured using fluorescence microscope (Olympus, USA) at $200 X$ magnification. A total of $\geq 200$ to 300 cells on four sub-grids of a haemocytometer was counted to determine the percentages of viable, apoptotic and necrotic cells.

\section{Gene expression}

The involvements of RAS, MEK1/2 and ERK1/2 in the underlying apoptotic signalling pathways induced by aloe emodin, tamoxifen and the combination of both in MCF-7 were determined using QuantiGene 2.0 multiplex assay (Affymetrix, USA). Ubiquitin C (UBC) and hypoxanthineguanine phosphoribosyltransferase (HPRT) were used as housekeeping genes (Affymetrix, USA). A total of $1 \times 10^{6}$ cells were treated with the same treatment groups as above, for up to 72 hours. Cell pellets were resuspended and kept at $-20^{\circ} \mathrm{C}$ before use. The assay was further conducted following the protocol, based on the series of hybridization methods that captured target RNA in the samples. They analyzed the expression of RNA through amplification of signal that can be detected by flow cytometry (I-DNA Biotechnology (M) Sdn. Bhd., Malaysia).

\section{Statistical analysis}

The differences between groups were evaluated using one-way ANOVA. Each experiment was repeated three times independently and in triplicates. Data obtained was expressed as the mean \pm standard deviation, $n=3$. Significance was set up at $p<0.05$.

\section{Results}

\section{Effects of aloe emodin and tamoxifen on the viability of MCF-7}

Previously, our group had determined the respective IC of aloe emodin and tamoxifen using WST-1 proliferation assay. Aloe emodin selectively inhibited the proliferation of ER $\alpha$-positive breast cancer MCF-7 cells with IC of $_{50} 80 \mu \mathrm{M}$ at 72 hours. No IC $\mathrm{I}_{50}$ was obtained for $E R \alpha$-negative breast cancer MDA-MB-231 and ER $\alpha$-negative non-transformed breast MCF-10A cells. In contrast, tamoxifen was nonselective to all three cells with $\mathrm{IC}_{50}$ of $27 \mu \mathrm{M}, 19 \mu \mathrm{M}$ and $38 \mu \mathrm{M}$, respectively (20). We further investigated the effect of aloe emodin and tamoxifen on MCF-7 cell viability using trypan blue exclusion test to reconfirm these findings. The representative microscopic images of aloe emodin, tamoxifen and combined treatments groups on MCF- 7 cell viability compared to untreated cells were shown in Figure 1A. Aloe emodin and tamoxifen inhibited the viability of MCF-7 cells by $32.00 \pm 1.19 \%$ to $43.18 \pm 3.21 \%$ ( $p<0.05$, $n=3)$ and $14.78 \pm 2.60 \%$ to $16.49 \pm 1.61 \%(p<0.05, n=3)$, respectively compared to untreated cells at 24 hours and up to 72 hours. Aloe emodin was a more potent inhibitor than tamoxifen $(p<0.05, n=3)$. Tamoxifen cytotoxicity on MCF-7 cells was significantly enhanced by aloe emodin with $22.05 \pm 1.20 \%, 37.50 \pm 2.23 \%$ and $36.93 \pm 2.19 \%$ ( $p<0.05$, $\mathrm{n}=3)$ increment at all treatment time points $(24,48$ and 72 hours) (Figure 1B). Aloe emodin and tamoxifen respective $\mathrm{IC}_{50}$ concentrations were also used in apoptosis and gene expression assays.

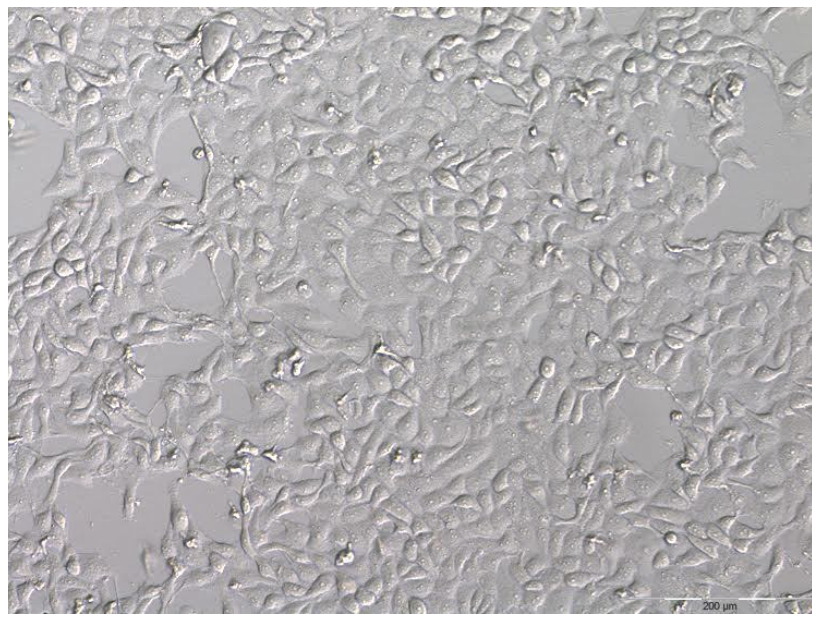

Figure 1A: Untreated image of MCF-7 cells

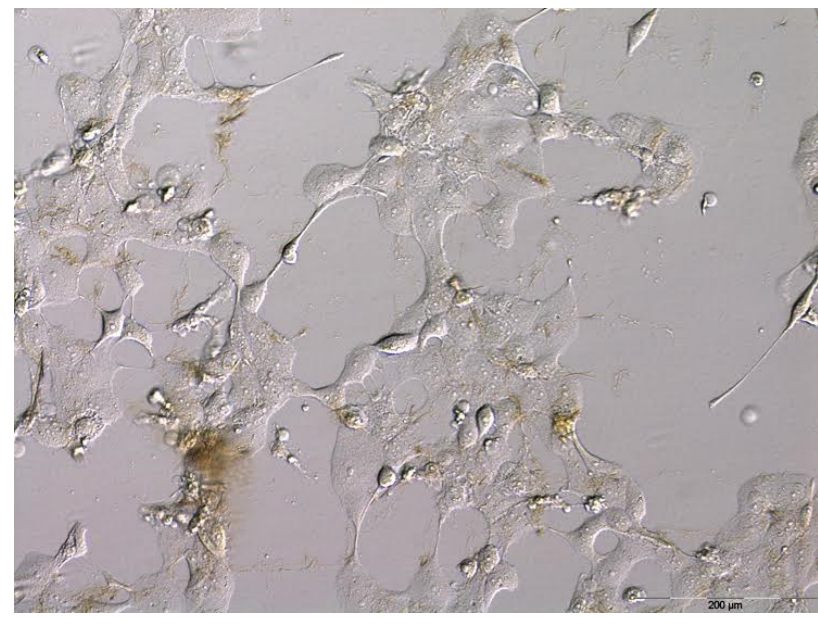

Figure 1A: Aloe emodin treated image 


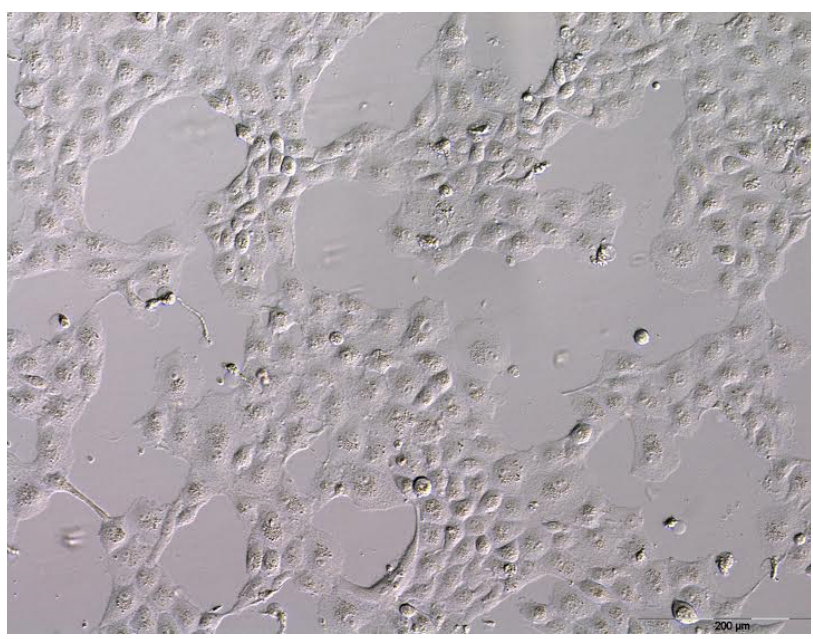

Figure 1A: Tamoxifen treated image

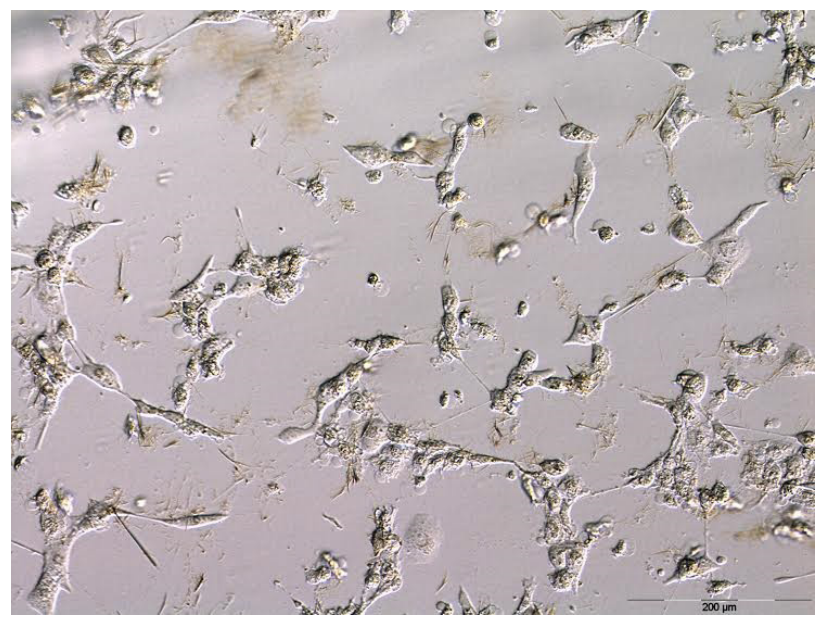

Figure 1A: Aloe emodin and tamoxifen treated image.

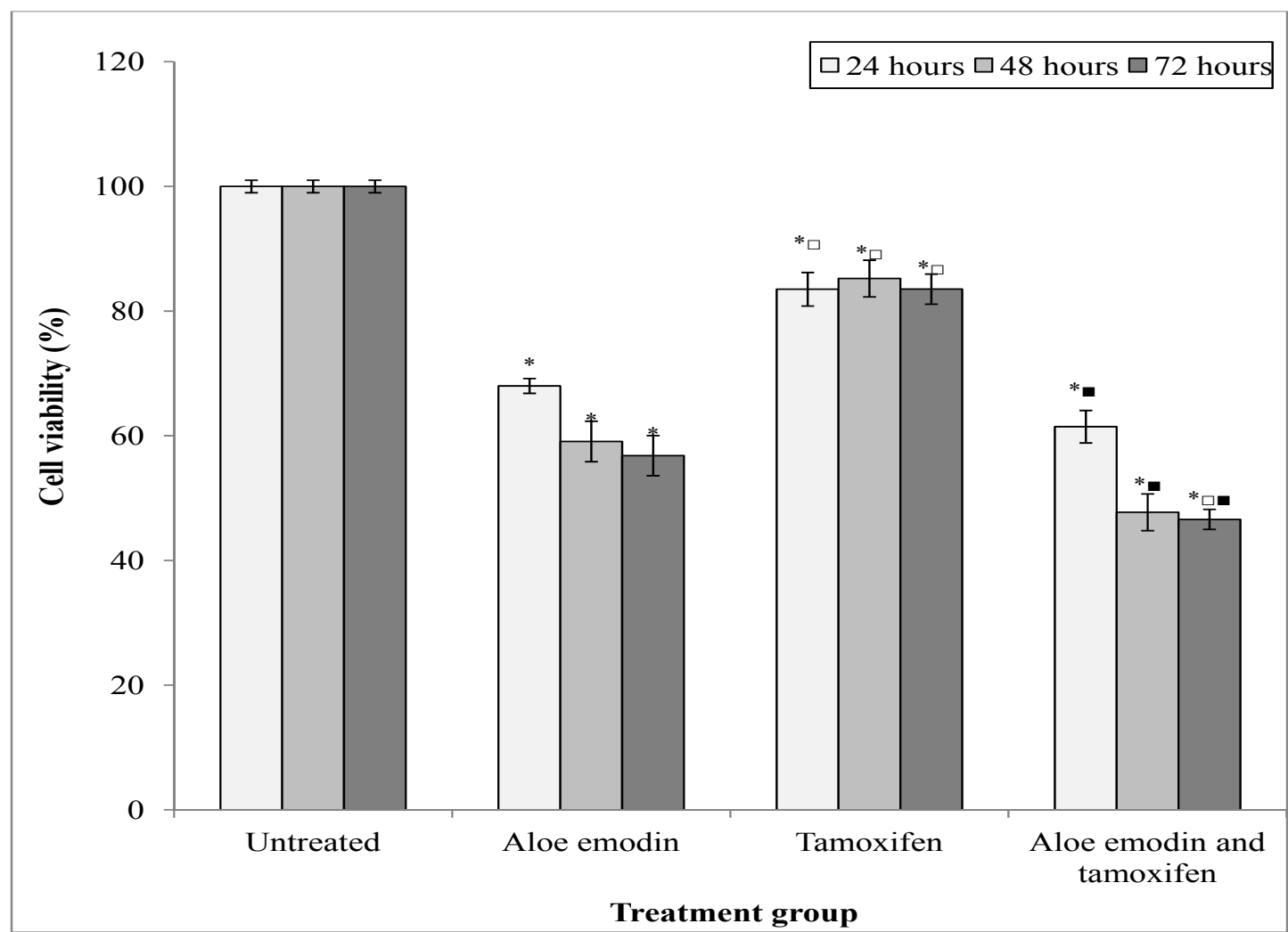

Figure 1B: Overall percentage of cell viability \pm SD obtained from three sets of experiments and in triplicates $(n=3)$ in a time-dependent manner

Figure 1: Cell viability effect of aloe emodin, tamoxifen and combination of both agents on MCF-7. The representative microscopic images of all three treatment groups compared to untreated cells (A). $4 \times 10^{5}$ cells were incubated with aloe emodin, tamoxifen and combination of both agents at respective $\mathrm{IC}_{50}$ up to 72 hours in $5 \% \mathrm{CO}_{2}$ at $37^{\circ} \mathrm{C}$. Cell morphology was observed under an inverted light microscope. The overall percentage of cell viability \pm SD obtained from three sets of experiments and in triplicates $(n=3)$ in a time-dependent manner is presented in bar graph (B). Aloe emodin is more potent as an inhibitory agent of MCF-7 viability compared to tamoxifen. Furthermore, tamoxifen cytotoxicity is enhanced by aloe emodin at all treatment time. *Significant as compared to untreated cells at $p<0.05$; ${ }^{\square}$ Significant as compared between aloe emodin with tamoxifen group at $p<0.05$; ${ }^{-}$Significant as compared between the combined treatment of aloe emodin and tamoxifen with tamoxifen alone in MCF-7 at $p<0.05$. 


\section{Effects of aloe emodin and tamoxifen on MCF-7 cell apoptosis}

The percentages of viable $(V)$, early apoptotic (EA), late apoptotic (LA) and necrotic (N) cells determined using $\mathrm{AO}$ and $\mathrm{PI}$ dual staining were represented by the images above (Figure 2A). A viable (V) cell appeared as green with intact nucleus. An early apoptotic (EA) cell was bright green from intercalation of both dyes in the nucleus. A late apoptotic (LA) cell was red/orange with the generation of the highest PI intensity emission. A necrotic $(\mathrm{N})$ cell was orange with disrupted cell membrane. The results were represented in Figure 2B.The presence of early and late apoptotic MCF-7 cells were seen after 48 hours of aloe emodin $(16.33 \pm 3.12 \%$ and $10.55 \pm 2.31 \%$; $p<0.05$,

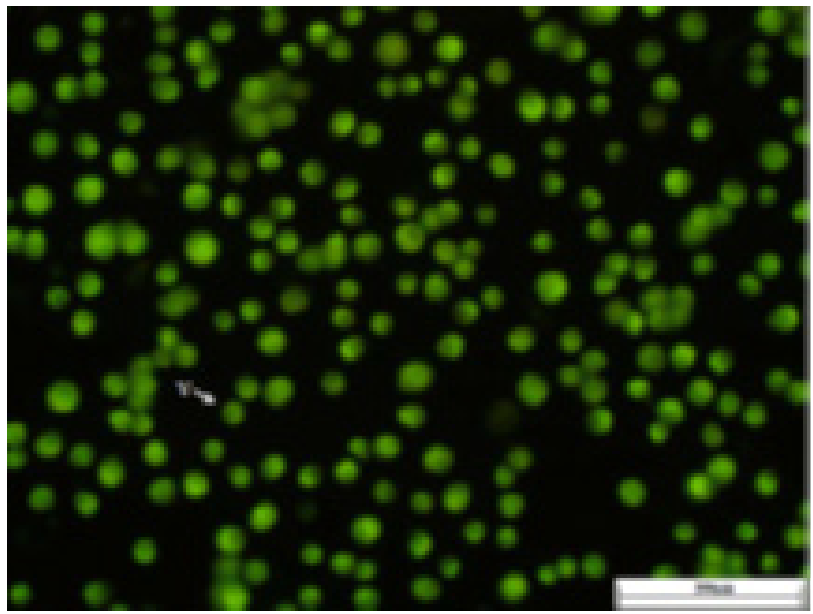

Figure 2A(a): Untreated MCF-7 cells image (48 hours)

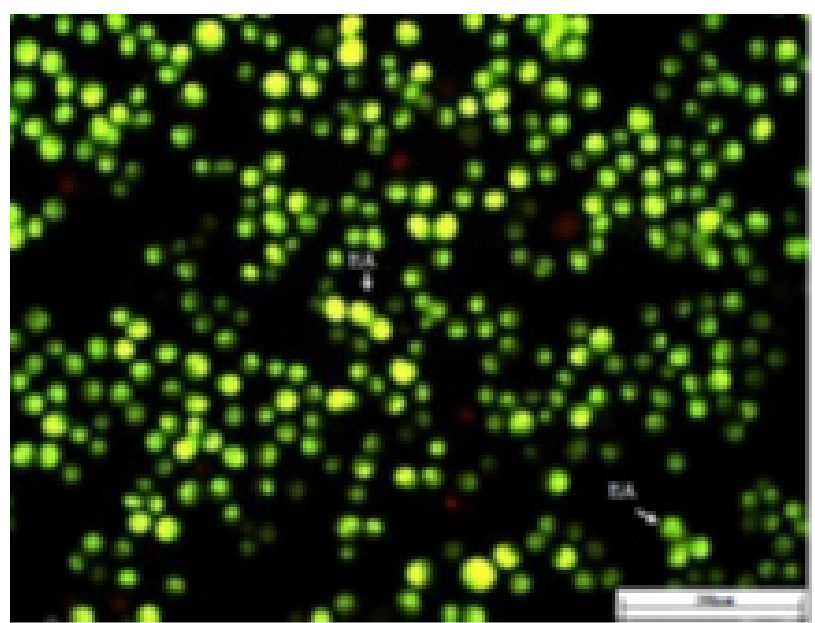

Figure 2A(a): Tamoxifen treated image (48 hours) $\mathrm{n}=3)$ and tamoxifen $(18.55 \pm 2.51 \%$ and $11.50 \pm 2.61 \%$; $\mathrm{p}<0.05, \mathrm{n}=3$ ) treatment compared to untreated cells. Increased apoptosis was seen in the combined treatment of aloe emodin with tamoxifen $(25.23 \pm 3.10 \%$ of early and $15.21 \pm 3.23 \%$ of late apoptosis; $p<0.05, n=3$ ) compared to untreated cells (Figure $\mathbf{2 B}(\mathbf{a})$ ). More cells underwent late apoptosis compared to early apoptosis after 72 hours of treatment. Higher apoptotic effect was also seen in the combined treatment of aloe emodin with tamoxifen $(25.32 \pm 2.50 \%$ of early and $50.22 \pm 2.51 \%$ of late apoptosis; $\mathrm{p}<0.05, \mathrm{n}=3)$ compared to aloe emodin $(15.52 \pm 3.25 \%$ of early and $35.31 \pm 2.23 \%$ of late apoptosis; $p<0.05, n=3$ ) and tamoxifen alone $(13.22 \pm 2.01 \%$ of early and $30.25 \pm 3.22 \%$ of late apoptosis; $p<0.05, n=3$ ) (Figure $2 B(b)$ ).

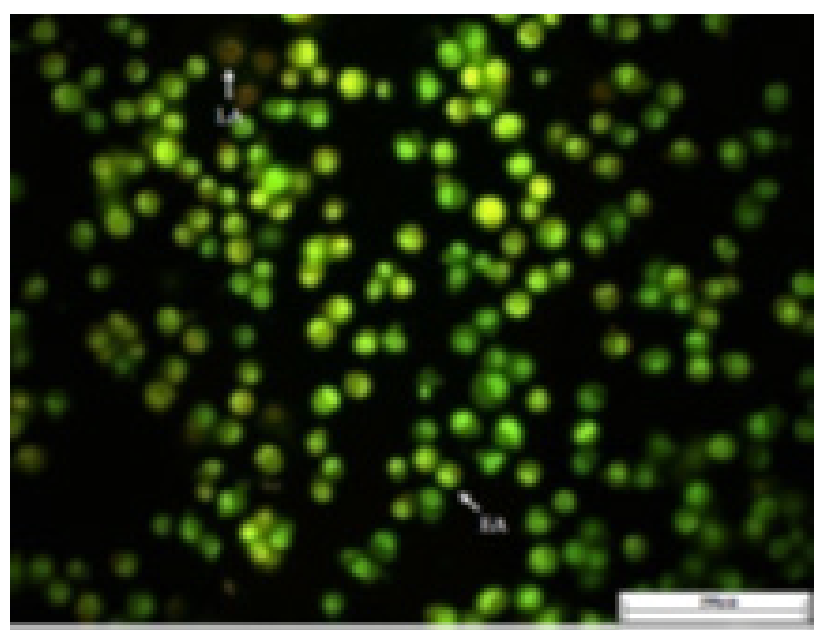

Figure 2A(a): Aloe emodin treated image (48 hours)

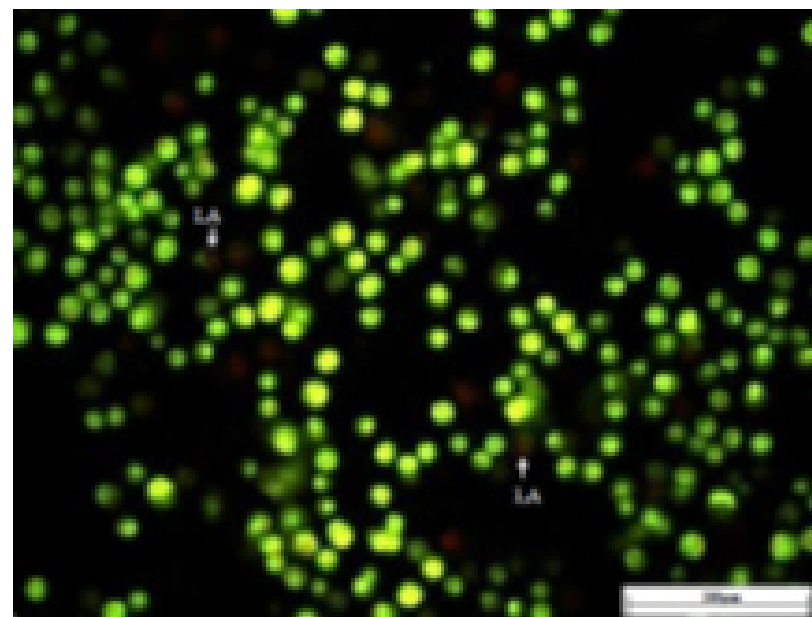

Figure 2A(a): Aloe emodin and tamoxifen treated image (48 hours) 


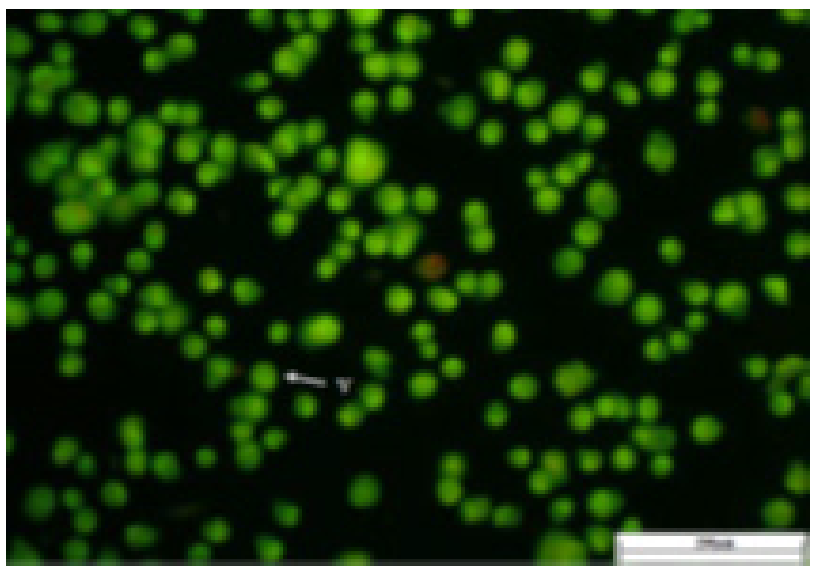

Figure 2A(b): Untreated MCF-7 cells image (72 hours)

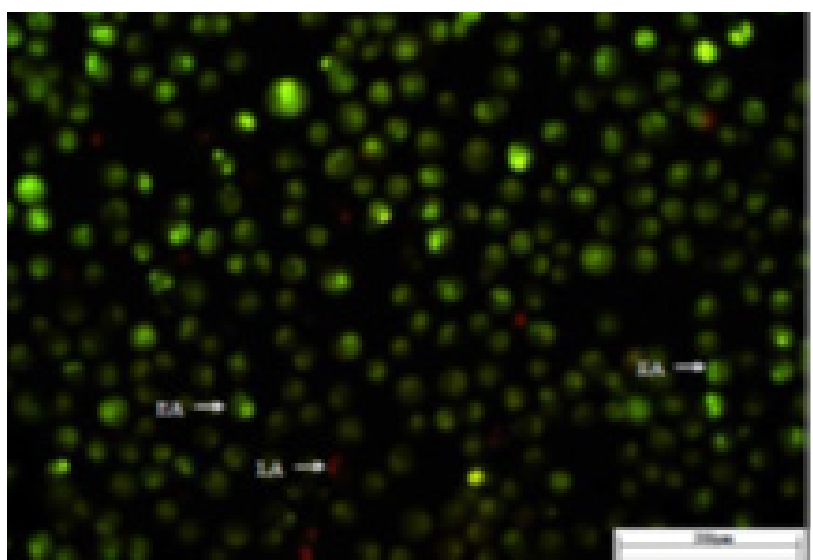

Figure 2A(b): Tamoxifen treated image (72 hours)

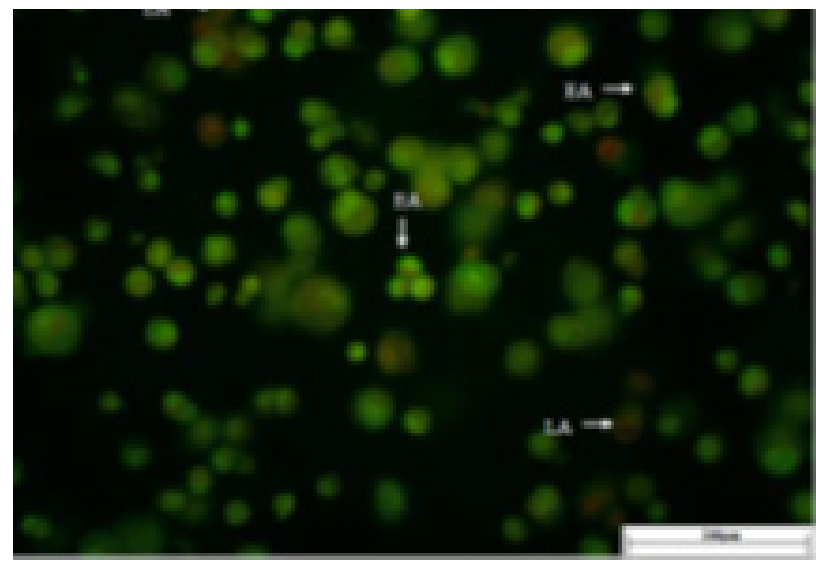

Figure 2A(b): Aloe emodin treated image (72 hours)

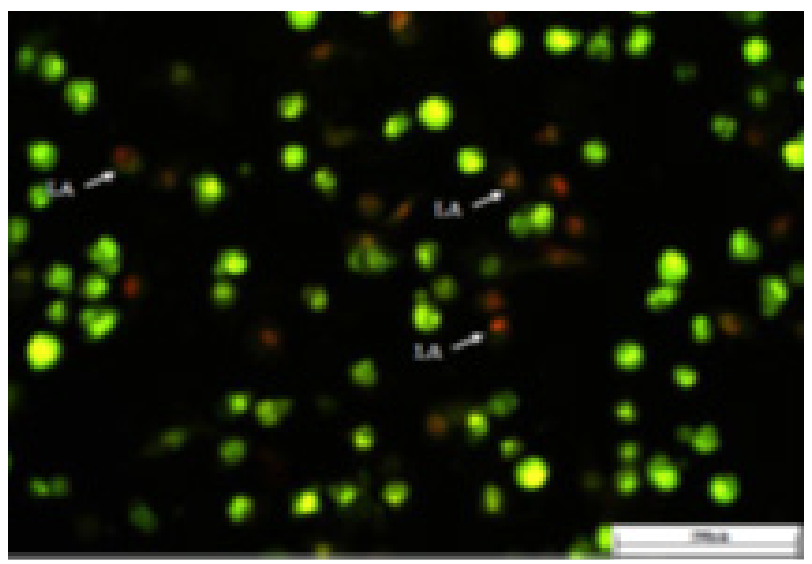

Figure $2 \mathrm{~A}(\mathrm{~b})$ : Aloe emodin and tamoxifen treated image (72 hours)

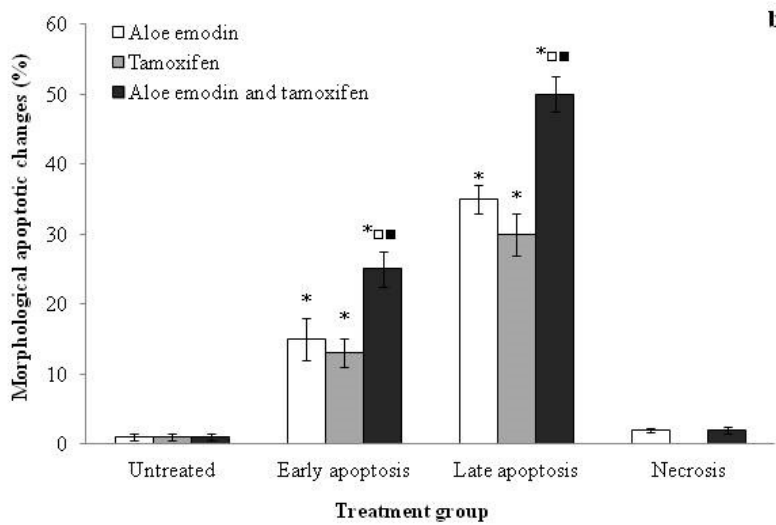

Figure $2 \mathrm{~B}(\mathrm{~b})$ :

Figure $2 \mathrm{~B}(\mathrm{a})$ :

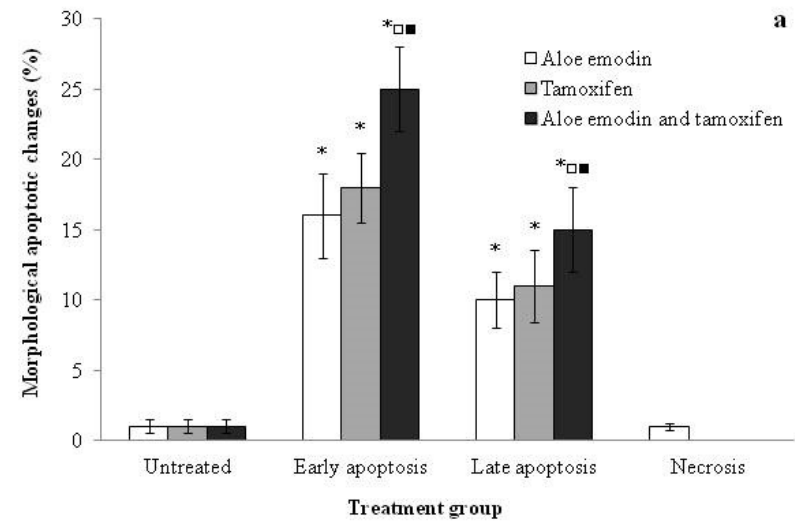

Figure 2: Apoptotic morphological changes of MCF-7 cells after treatment with aloe emodin, tamoxifen and combination of both agents. $4 \times 10^{5}$ cells were treated with aloe emodin, tamoxifen and combination of both agents at respective $\mathrm{IC}_{50}$ up to 72 hours in $5 \%$ $\mathrm{CO}_{2}$ at $37^{\circ} \mathrm{C}$. Apoptotic morphological changes were observed by applying AO and PI dual staining. Cells were identified as viable (V), early apoptosis (EA), late apoptosis (LA) and necrosis $(N)$ after 48 hours and 72 hours of treatment represented by Figure $2 \mathrm{~A}(\mathrm{a})$ and $2 A(b)$, respectively. The percentage of these changes obtained from all three sets of experiments and in triplicate $(n=3)$ at different times ( 48 hours and 72 hours) was represented in the Figure $2 \mathrm{~B}(\mathbf{a})$ and Figure $\mathbf{2 B}(\mathbf{b})$, respectively. At 48 hours, higher apoptosis was seen in aloe emodin and tamoxifen combined treatment in MCF-7 cells compared to aloe emodin and tamoxifen alone (Figure 2B(a)). Similar pattern was seen at 72 hours ((Figure $\mathbf{2 B}(\mathbf{b})$ ). *Significant as compared to untreated cells at $\mathrm{p}<0.05$; ${ }^{\square}$ Significant as compared

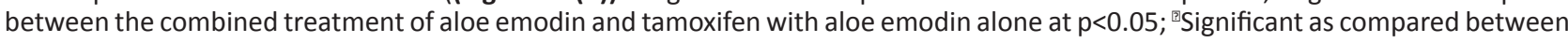
the combined treatment of aloe emodin and tamoxifen with tamoxifen alone at $p<0.05$. 


\section{Effects of aloe emodin and tamoxifen on the RAS, MEK1/2 and ERK1/2 signalling of MCF-7}

The effect of aloe emodin and tamoxifen-combined treatment on the expression of RAS, MEK1/2 and ERK1/2 genes in the MAPK proliferation pathway of MCF-7 was determined. UBC housekeeping gene was used to normalize the expression of target genes. Similar pattern was seen using HPRT gene. All the target genes were downregulated by the respective aloe emodin and tamoxifen treatment in MCF-7 (Figure 3). Synergistic effect was seen in MEK1/2 genes (Figure 3B).

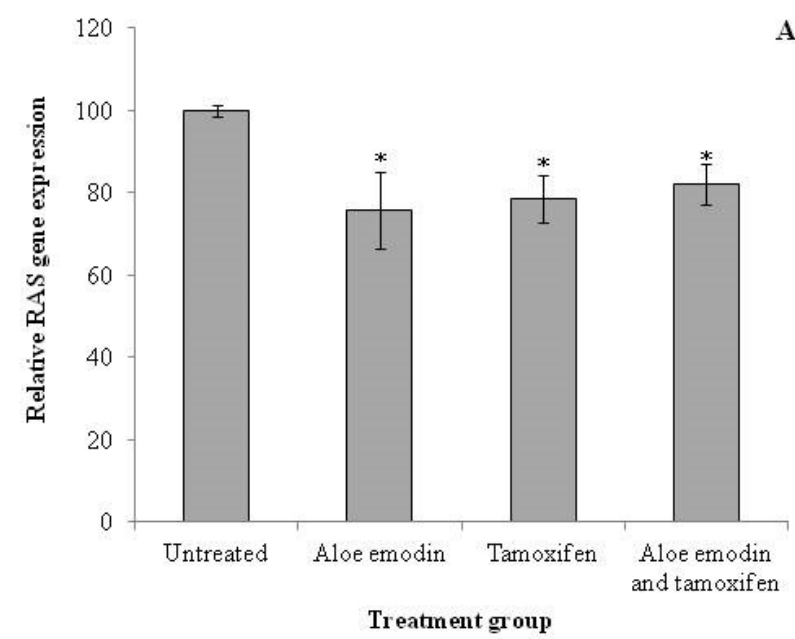

Figure 3A

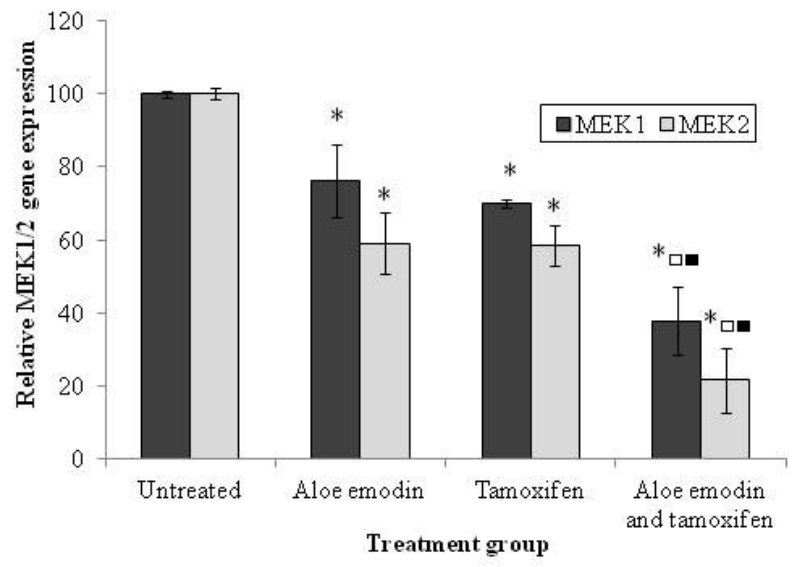

Figure 3B

C

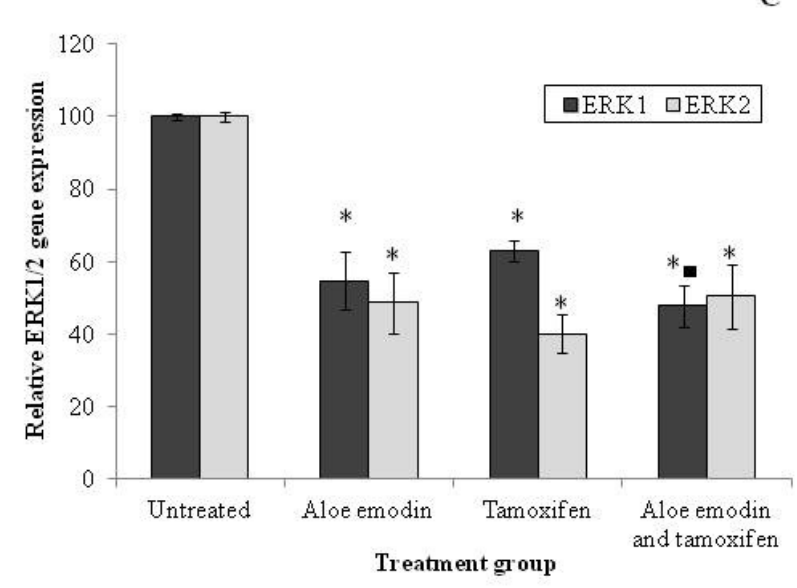

Figure 3C

Figure 3: RAS (A), MEK1/2 (B) and ERK1/2 (C) gene expression of MCF-7 cells after treatment with aloe emodin, tamoxifen and combination of both agents. $1 \times 106$ cells were treated aloe emodin, tamoxifen and combination of both agents at respective IC50 up to 72 hours in $5 \% \mathrm{CO} 2$ at $370 \mathrm{C}$. The assay was conducted following the QuantiGene 2.0 multiplex assay. The mRNA level of all target genes was expressed as \% normalized to UBC housekeeping gene \pm SD. The experiments were duplicated and repeated thrice $(n=3)$. Similar pattern was seen using HPRT as housekeeping gene. Higher downregulation of MEK1/2 genes expression were seen in the combined treatment compared to aloe emodin and tamoxifen alone, respectively $(p<0.05)$. *Significant as compared to untreated cells at $p<0.05$; $\square$ Significant as compared between the combined treatment of aloe emodin and tamoxifen with aloe emodin alone at $p<0.05$; Significant as compared between the combined treatment of aloe emodin and tamoxifen with tamoxifen alone at $p<0.05$. 


\section{Discussion}

Aloe emodin, a hydroxyanthraquinone from Aloe barbadensis Miller leaves, has been found to have anticancer effects on several cancer cell lines such as Merkel and prostate $(17,18)$. It is non-toxic as demonstrated in an in vivo model (21). The effect of aloe-emodin on ER $\alpha$ positive breast cancer cells is not well understood. In the previous study, we clearly demonstrated the selective anti cancer activity of aloe emodin on $\mathrm{ER} \alpha$-positive breast cancer cells, MCF-7 but not on ER $\alpha$-negative breast cancer, MDA-MB-231 and ER $\alpha$-negative non-transformed breast cells, MCF-10A (20). Unlike tamoxifen, aloe emodin was selectively cytotoxic for MCF-7 breast cancer cells (20). The inhibitory effect of Aloe emodin and tamoxifen on MCF-7 at $\mathrm{IC}_{50}$ of $80 \mathrm{mM}$ which had been published previously (20) was reconfirmed by trypan blue exclusion test. A combination treatment of aloe emodin with tamoxifen further inhibited the viability of MCF-7, reflecting an enhancement in the cytotoxicity effect significantly with $\mathrm{p}<0.05$ (Figure 1B). To examine the mechanism responsible for cell viability inhibition by both treatments, morphological apoptotic characteristics were evaluated. The findings suggested that cytotoxicity of aloe emodin and tamoxifen on MCF-7 cells was through apoptosis, not necrosis. An enhancement in apoptotic effect was seen in the combination treatment (Figure 2A) as represented by Figure 2B. Chen et al (22) showed a cytotoxicity effect using aloe emodin-loaded solid lipid nanoparticles (AE-SLNs) on MCF-7, but not on MCF-10A. AE-SLNs were prepared in an attempt to improve the anti-cancer efficacy of aloe emodin (22). It exhibited excellent cytotoxicity against MCF-7 but not on MDA-MB-453 cells (15). Aloe emodin was selectively active against neuroectodermal tumour cells in both in vitro and in vivo models, and induced cell shrinkage, membrane blebbing and nuclear fragmentation; but not in human hematopoietic progenitors and normal fibroblasts (21). The hematopoietic cells were not affected at concentrations more than 100 times higher than those required to inhibit neuroectodermal tumour cell growth (21). Lymphoid cells (JURKAT and CCRF-CEM) and the most resistant of myeloid cells (KG-1a and K562) were responsive to aloe emodin through induction of apoptosis, but not in non-tumour cells (23). Similar effects were seen with its isomer, emodin (1,3,8-trihydroxy-6-methylanthraquinone) (23). Both compounds produced a synergistic effect when combined with etoposide and doxorubicin on both lymphoid cell lines (24). The combination of aloe emodin and radiation enhanced radiosensitivity and apoptosis of cervical cancer cells, HeLa, compared to treatment with aloe emodin or radiation alone (25). Co-treatment of emodin, with cisplatin significantly elevated ROS levels and enhanced the chemosensitivity in prostatic cancer DU-145 cells, compared with cisplatin alone. The combination therapy had little effect on normal human dermal fibroblasts (18). These evidences showed that aloe emodin and emodin had a potential role of enhancing the action of chemotherapy in cancer treatment. A significant regression was seen among patients with metastatic solid tumours. These tumours included lung cancer treated with cisplatin and etoposide, colorectal cancer on oxaliplatin and 5-fluorouracil; gastric cancer on 5-fluorouracil, and pancreatic cancer receiving gemcitabine. The patients were randomised to a group concomitantly treated with Aloe arborescens extracts (10mL thrice/daily orally) and a group on chemotherapy alone. An increment in the percentage of a 3-year survival rate was also noted (26). Further study to investigate the possible signalling pathways is required.

The MAPKs signalling pathway is a key intracellular cascade in the regulation of normal cell proliferation, cell cycle, survival, angiogenesis and migration. Alteration in the family components particularly the ERKs as survival protein cascades contribute to cancer and other human diseases (7). It has been the focus of interest in the development of pharmacologic inhibitors for the treatment of cancer (6-8). To elucidate whether the expressions of RAS, MEK1/2 and ERK1/2 are involved in aloe emodin-induced apoptosis in MCF-7 cells, we examined the expression of these genes using QuantiGene 2.0 multiplex techniques during aloe emodin-mediated apoptosis. Exposure of MCF-7 cells to aloe emodin at IC $C_{50}$ resulted in decreased RAS, MEK1/2 and ERK1/2 expressions after 72 hours of treatment, similar to tamoxifen. Combination of aloe emodin and tamoxifen significantly reduced the expression of MEK1/2 with $p<0.05$ (Figure 3B). Aloe emodin also induced apoptosis in lung non-small carcinoma cells, $\mathrm{H} 460$, through inactivation of the ERK signalling pathway (27). Elevated levels of Rad51 expression seen in chemo or radioresistant lung carcinomas are decreased in nonsmall-cell lung cancer cells, H1703 and A549 by the synergistic effect of emodin (Rheum palmatum $L$ root and rhizome extracts) with anti-tumor antibiotic mitomycin $C$ through inactivation of ERK pathway (28). It enhanced the cytotoxicity effect of cisplatin in advanced non-small cell lung cancer cells, (NSCLC) through the downregulation of excision repair cross-complementation 1 (ERCC1), an enzyme crucial for the removal of adducts from genomic DNA, and inactivation of ERK survival pathway (29). Alizarin (1,2-dihydroxyanthraquinone), another anthraquinone from madder root extract inhibited osteosarcomas such as Saos-2, MG-63 and U-2 OS cells at lower doses compared to normal cells, demonstrating a selective activity towards malignant cells. It acted through the inhibition of ERK1/2 phosphorylation and S phase arrest (30).

The data obtained from this study is useful for future work on the apoptotic effects of aloe emodin at protein level.

\section{Conclusion}

Our findings are in line with published work which showed that natural anthraquinone selectively inhibited the proliferation of cancer cells at a lower dose compared to the normal cells, by regulating the ERK signalling pathway. We showed that aloe emodin enhanced tamoxifen cytotoxicity on ER $\alpha$-positive breast cancer, MCF-7 cells, was mediated by MEK1/2 downregulation. These findings may provide a basis for the therapeutic usage of aloe emodin to enhance the efficacy of chemotherapy in ER $\alpha$-positive breast cancer patients. 


\section{Acknowledgements}

This research was funded by Universiti Teknologi MARA, Malaysia (600-RMI/ST/DANA 5/3/Dst (304/2009)) and Ministry of Higher Education (MOHE), Malaysia (600-RMI/ RAGS 5/3 (86/2013).

\section{Competing interests}

The authors declare that they have no competing interests.

\section{References}

1. Pathya NB, Yip HC, Taib NA, Hartman M, Saxena N, Lau $P$, et al. Malaysian breast cancer working group, Breast cancer in a multi-ethnic Asian setting: Results from the Singapore Malaysia hospital-based breast cancer registry. The Breast 2011; 20:75-80.

2. Abdullah NA, Wan Mahiyuddin WR, Muhammad NA, Mohamad Ali Z, Ibrahim L, Ibrahim Tamim NS, et al. Survival rate of breast cancer patients in Malaysia: A population-based study. Asian Pac J Cancer Prev. 2013; 14(8):4591-4.

3. Yamashita, H. Current research topics in endocrine therapy for breast cancer. Int J Clin Oncol. 2008; 13(5):380-3.

4. Haagenson KK, Wu GS. The role of MAP kinases and MAP kinase phosphatase-1 in resistance to breast cancer treatment. Cancer Metastasis Rev. 2010; 29(1):143-9.

5. Karn A, Jha AK, Shrestha S, Acharya B, Poudel S, Bhandari RB. Tamoxifen for breast cancer. J Nepal Med Assoc. 2010; 49(177):62-7.

6. Kurebayashi J, Kanomata N, Kozuka Y, Moriya T, Kikukawa N, Kawasaki Y, et al. The cell cycle profile test is a prognostic indicator for breast cancer patients treated with postoperative 5-fluorouracil-

based chemotherapy. J Clin Oncol. 2011; 41(6):739-6.

6. Reddy KB, Nabha SM, Atanaskova N. Role of MAP kinase in tumor progression and invasion. Cancer Metastasis Rev 2003; 22:395-403.

7. Holdefield M, Merritt $H$, Chan J, Wallroth M, Tandeske $\mathrm{L}$, Zhai $\mathrm{H}$, et al. RAF inhibitors activate the MAPK pathway by relieving inhibitory autophosphorylation. Cancer Cell. 2013; 23(5):594-602.

8. McCubrey JA, Steelman LS, Chappell WH, Abrams SL, Wong EW. Chang F et al. Roles of the Raf/MEK/ERK pathway in cell growth, malignant transformation and drug resistance. Biochim Biophys Acta. 2007; 1773(8):1263-84.

9. Harada H, Quearry B, Ruiz-Vela A, Korsmeyer SJ. Survival factor induced extracellular signal-regulated kinase phosphorylates BIM, inhibiting its association with BAX and proapoptotic activity. Proc Natl Acad Sci USA 2004; 101:15313-7.

10. Allan LA, Morrice N, Brady S, Magee G, Pathak $S$, Clarke PR. Inhibition of caspase-9 through phosphorylation at Thr 125 by ERK MAPK. Nat Cell Biol 2003; 7:647-54.
11. Nicholson $R I$, Hutcheson $I R$, Britton $D$, Knowlden $\mathrm{JM}$, Jones $\mathrm{HE}$, Harper $\mathrm{ME}$, et al. Growth factor signalling networks in breast cancer and resistance to endocrine agents: new therapeutic strategies. J Steroid Biochem Mol Biol Cancer Res 2005; 93:257-62.

12. Britton DJ, Hutcheson IR, Knowlden JM, Barrow D, Giles $M$, McClelland RA, et al. Bidirectional cross talk between ER $\alpha$ and EGFR signalling pathways regulates tamoxifen-resistant growth. Breast Cancer Res Treat 2006; 96:131-46.

13. Chan DW, Hui WW, Cai PC, Liu MX, Yung MM, Mak CS, et al. Targeting GRB7/ERK/FOXM1 signalling pathway impairs aggressiveness of ovarian cancer cells. PLOS One._2012; 7(12):1-10.

14. Jin F, Irshad S, Yu W, Belakavadi M, Chekmareva $M$, Ittmann MM, et al. ERK and AKT signalling drive MED1 overexpression in prostate cancer in association with elevated proliferation and tumorigenicity. Mol Cancer Res. 2013; 11(7):736-47.

15. Huang $\mathrm{PH}$, Huang $\mathrm{CY}$, Chen MC, Lee YT, Yue $\mathrm{CH}$, Wang HY, et al. Emodin and aloe-emodin suppress breast cancer cell proliferation through ER $\boldsymbol{\alpha}$ inhibition. JEBCAM. 2013; 2013:1-12.

16. Patisaul HB, Jefferson W. The pros and cons of phytoestrogens. Front Neuroendocrinol. 2010; 31(4):400-19.

17. Fenig E, Nordenberg J, Beery E, Sulkes J, Wasserman L. Combined effect of aloe-emodin and chemotherapeutic agents on the proliferation of an adherent variant cell line of Merkel cell carcinoma. Oncol Rep. 2004; 11(1):213-7.

18. Huang $X Z$, Wang J, Huang C, Chen YY, Shi GY, $\mathrm{Hu} \mathrm{QS}$, et al. Emodin enhances cytotoxicity of chemotherapeutic drugs in prostate cancer cells: the mechanisms involve ROS-mediated suppression of multidrug resistance and hypoxia inducible factor-1. Cancer Biol Ther 2008; 7:468-75.

19. Strober W. Trypan blue exclusion test of cell viability. $1^{\text {st }}$ ed. John Wiley and Sons, Inc; 2001.

20. Amin IM, Sheikh Abdul Kadir SH, Nik Mohd Rosdy NMM, Siran R, Hasani NAH. Anti-cancer effect of aloe emodin on breast cancer cells, MCF-7. Proceedings of the 7th International Conference on Systems Biology (ISB), IEEE Xplore Press, China 2013; 103-108.

21. Pecere T, Gazzola MV, Mucignat C, Parolin C, Vecchia FD, Cavaggioni A, et al. Aloe-emodin is a new type of anticancer agent with selective activity against neuroectodermal tumors. Cancer Res 2000;60:2800-4.

22. Chen R, Wang S, Zhang J, Chen M, Wang Y. Aloe-emodin loaded solid lipid nanoparticles: formulation design and in vitro anti-cancer study. Drug Deliv 2014:1-9.

23. Mahbub AA, Le Maitre CL, Haywood-Small SL, McDougall GJ, Cross NA, Jordan Mahy N. Differential effects of polyphenols on proliferation and apoptosis in human myeloid and lymphoid leukemia cell lines. Anticancer Agents Med Chem. 2013; 13(10):1601-13.

24. Mahbub AA, Le Maitre CL, Haywood-Small SL, McDougall GJ, Cross NA, Jordan Mahy N. Quercetin 
and emodin synergistically enhanced chemotherapy activity in human lymphoid and myeloid leukaemia cell lines, in vitro. 4th International Congress on Leukemia-Lymphoma-Myeloma, Turkey 2013; 275.

25. Luo J, Yuan Y, Chang P, Li D, Liu Z, Qu Y. Combination of aloe-emodin with radiation enhances radiation effects and improves differentiation in human cervical cancer cells. Mol Med Rep 2014; 10:731-6.

26. Lissoni P, Rovelli F, Brivio F, Zago R, Colciago M, Messina $G$, et al. A randomized study of chemotherapy versus biochemotherapy with chemotherapy plus Aloe arborescens in patients with metastatic cancer. In Vivo. 2009; 23(1):171-5.

27. Yeh $\mathrm{FT}, \mathrm{Wu} \mathrm{CH}$, Lee $\mathrm{HZ}$. Signalling pathway for aloeemodin-induced apoptosis in human $\mathrm{H} 460$ lung nonsmall carcinoma cell. Int. J. Cancer 2003; 106:26-33.
28. Su YJ, Tsai MS, Kuo YH, Chiu YF, Cheng CM, Lin ST, et al. Role of Rad51 down-regulation and extracellular signal-regulated kinases 1 and 2 inactivation in emodin and mitomycin C-induced synergistic cytotoxicity in human non-small-cell lung cancer cells. Mol Pharmacol. 2010; 77(4):633-43.

29. Ko JC, Su YJ, Lin ST, Jhan JY, Ciou SC, Cheng CM, et al. Emodin enhances cisplatin-induced cytotoxicity via down-regulation of ERCC1 and inactivation of ERK1/2. Lung Cancer. 2010; 69(2):155-64.

30. Fotia C, Avnet S, Granchi D, Baldini N. The natural compound Alizarin as an osteotropic drug for the treatment of bone tumors. J Orthop Res. 2012; 30(9):1486-92. 